\title{
Bone Marrow Mesenchymal Stem Cells-Derived Exosomes Upregulate MicroRNA-424-5p to Suppress Hepatocellular Carcinoma Cell Growth by Inhibiting Forkhead Box K1
}

\section{Zewei Lin}

Shenzhen Hospital, Peking University

\section{Qingqi Ren}

Shenzhen Hospital, Peking University

Xiaofei Ma

Shenzhen Hospital, Peking University

Liu Jikui ( $\square$ liujikui8929@126.com )

Shenzhen Hospital, Peking University https://orcid.org/0000-0002-0824-9404

\section{Research Article}

Keywords: Hepatocellular carcinoma, Bone marrow mesenchymal stem cells-derived exosome, MicroRNA424-5p, Forkhead box K1

Posted Date: April 30th, 2021

DOI: https://doi.org/10.21203/rs.3.rs-453742/v1

License: (c) (i) This work is licensed under a Creative Commons Attribution 4.0 International License. Read Full License 


\section{Abstract}

Bone marrow mesenchymal stem cells (BM-MSCs) have been shown to exert a potential therapeutic effect during tumor treatment and it has been proved that exosomes derived from BM-MSCs play crucial roles in the progression of malignant tumors. The current study aims to investigate the effect of BM-MSCderived exosomal microRNA-424-5p (miR-424-5p) on hepatocellular carcinoma (HCC) progression. The expression of miR-424-5p was compared between HCC and adjacent normal tissues, and its prognosis value was analyzed. Additionally, exosomes were extracted from the BM-MSCs and their identity was verified. Luciferase reporter assay was conducted to identify the putative binding sites between miR-424$5 p$ and the 3'-UTR of forkhead box K1 (FOXK1). The BM-MSC-derived exosomes were co-cultured with HCC cells to assess the effect of the BM-MSC-derived exosomes, miR-424-5p, and FOXK1 on the proliferation, migration, invasion, and in vivo tumorigenesis of the HCC cells. Then, the expression of FOXK1 was also examined in HCC and normal tissues. miR-424-5p was downregulated and FOXK1 was upregulated in HCC tissues and cells. BM-MSC-derived exosomes upregulated miR-424-5p expression to suppress the proliferation, migration, invasion, and in vivo tumorigenesis of HCC cells. Knockdown of FOXK1 also repressed the malignant behavior of the HCC cells, and FOXK1 was verified as the target of miR-424-5p. The role of FOXK1 silencing in HCC cells was reversed by miR-424-5p downregulation. Our results suggested that BM-MSC-derived exosomes upregulated miR-424-5p expression to restrain HCC cell growth and invasion via inhibition of FOXK1 expression and as a result, decelerating HCC development.

\section{Introduction}

Liver cancer is the 6th most common type of cancer and approximately 841,000 new cases are diagnosed and 782,000 deaths occur each year ${ }^{1,2}$. HCC accounts for about $80 \%$ of primary liver cancers and is the main pathological type ${ }^{2}$. HCC is a leading cause of cancer-related death worldwide ${ }^{3}$. The prognosis of HCC patients is poor due to the limited number of therapeutic methods available for advanced $\mathrm{HCC}^{4}$. All therapeutic options, such as surgery with adjuvant chemotherapy or radiotherapy, are typically only available for those with early-stage $\mathrm{HCC}^{5-7}$. Therefore, there is an urgent need to explore potential biomarkers for HCC, elucidate mechanisms implicated in HCC carcinogenesis, and development for the evaluation of molecular therapeutic targets.

BM-MSCs are non-hematopoietic stem cells found in bone marrow that have the ability of self-renewal, pronounced proliferation, and differentiation into various other types of cells ${ }^{8,9}$. Exosomes are small (diameter of 30-100 nm) membrane-bound vesicles secreted by multiple cell types, and it has been reported that exosomes show tremendous potential for the effective treatment of $\mathrm{HCC}^{10-12}$. However, the role of BM-MSC-derived exosomes in HCC remains largely unknown. miRNAs are small non-coding RNAs that repress gene expression by binding to the 3 '-untranslated region (3'-UTR) to influence mRNA stability or protein translation ${ }^{13,14}$. Specific miRNAs, such as miR-342-3p ${ }^{15}$, miR- $612^{16}$, and miR-541 ${ }^{17}$, have been confirmed to suppress HCC cell growth. Importantly, it has been verified that miR-424-5p acts as a 
potential biomarker of $\mathrm{HCC}^{18}$. Nevertheless, the role of exosomal miR-424-5p, especially BM-MSC-derived exosomal miR-424-5p in HCC remains scarcely investigated. The FOXK family is a subgroup of forkhead transcripts and is known to modulate various biological processes, such as metabolism, survival, apoptosis, differentiation, and senescence ${ }^{19-21}$. As a member of the FOXK family, FOXK1 has been confirmed to be correlated with a poor prognosis of HCC and regulates the stemness of HCC cells ${ }^{22}$. It has also been verified that FOXK1 knockdown was able to suppress HCC cell growth ${ }^{23}$. However, the relationship between miR-424-5p and FOXK1 is still unknown.

We aimed to investigate the effect of BM-MSC-derived exosomal miR-424-5p on the biological processes of HCC cells by targeting FOXK1, and we speculated that BM-MSC-derived exosomes may regulate miR424-5p expression and thus affect HCC cell growth through the involvement of FOXK1.

\section{Materials And Methods}

\section{Patients and tissue samples}

Ninety pairs of primary HCC tissue and adjacent non-tumor liver tissue $(>2 \mathrm{~cm}$ and $<5 \mathrm{~cm}$ from tumor edge) were collected from patients (56 males and 41 females; age, 41-73 years; mean age, 52.6 years) who underwent surgical resection at our hospital from October 2013 to January 2015. The tissue samples were immediately frozen and preserved at $-80^{\circ} \mathrm{C}$ in liquid nitrogen. None of the patients suffered from hepatitis or had previously accepted anti-inflammatory treatment or oncotherapy.

Written informed consent was obtained from all patients before the study and the diagnosis of all tissue samples was made and independently confirmed by two pathologists. Overall survival was calculated during the duration between surgery and death or last follow-up. This study was approved by the Shenzhen Hospital, Peking University Institutional Ethics Committee and conducted following Ethical Principles for Medical Research Involving Human Subjects of the Helsinki Declaration.

\section{Cell culture}

HCC cell lines (HL7702, HCCLM3, HepG2, and MHCC97-H) and human immortalized hepatocyte line L02, and human BM-MSCs were obtained from the American Type Culture Collection. The cells were all cultured with DMEM containing 10\% fetal bovine serum and $1 \%$ penicillin-streptomycin.

\section{Flow cytometry identification of the BM-MSCs}

Specific stem cell markers were detected using flow cytometry, as previously described ${ }^{24,25}$. In brief, BMMSCs were harvested and washed. Then, Phycoerythrin (PE)-labeled anti-CD34, CD44, CD45, CD90, and the isotype control was incubated with the BM-MSCs for 20 min. After washing with PBS, the BM-MSCs were examined using flow cytometry.

\section{Isolation and identification of the BM-MSC-derived exosomes}


Exosomes were extracted from the culture supernatant of the BM-MSCs using ultracentrifugation and their identity was confirmed using previously reported methods ${ }^{26,27}$. In brief, the BM-MSCs were cultured in DMEM containing $10 \%$ FBS (FBS was pre-centrifuged at $16,000 \times \mathrm{g}$ for 60 min to remove the exosomes). After $24 \mathrm{~h}$ of culture, the medium supernatant was centrifuged at $16,000 \times \mathrm{g}$ for $60 \mathrm{~min}$ at $4^{\circ} \mathrm{C}$ and the exosomes were harvested.

\section{Cell transfection}

The cells were plated and cultured at $37^{\circ} \mathrm{C}$ for $24 \mathrm{~h}$. Next, the cells were transfected with miR-424-5p mimic, miR-424-5p inhibitor, siRNA-FOXK1, or the NC, respectively, using a Lipofectamine 2000 system, following the manufacturer's instructions. Transfection efficiency was measured after $48 \mathrm{~h}$.

\section{PKH26-labeled exosomes}

For exosome labeling, a PKH26 kit was used as instructed by the manufacturer. In brief, exosomes were suspended in Diluent $\mathrm{C}$ and then mixed with PKH26. After incubation for 20 minutes in the dark at room temperature, the labeling reaction was stopped and the labeled exosomes were ultra-centrifuged at $100,000 \times \mathrm{g}$ for $60 \mathrm{~min}$. After washing with PBS, the exosomes were again ultra-centrifuged at 100,000 $\times \mathrm{g}$ for $60 \mathrm{~min}$ and resuspended in a serum-free medium, and co-cultured with the HCC cells. Then, the labeled exosomes were observed under a confocal laser scanning microscope.

\section{Transmission electron microscopy}

The isolated exosomes were fixed in $200 \mu \mathrm{L}$ of $3 \%$ glutaraldehyde for $100 \mathrm{~min}$. A drop (20 $\mu \mathrm{L})$ of exosomes was transferred onto a formvar-carbon-coated grid. After rinsing, the grids were stained with $1 \%$ uranyl acetate solution for $5 \mathrm{~min}$. Then, the grids were washed twice and examined under a transmission electron microscope at $80 \mathrm{kV}$.

\section{Exosome uptake experiment}

MHCC97-H cells were incubated with the BM-MSC-derived exosomes and stained with $1 \mathrm{~mL}$ of 4',6diamidino-2-phenylindole $2 \mathrm{HCl}$ for $10 \mathrm{~min}$. BM-MSC uptake by the MHCC97-H cells was observed under a fluorescence microscope.

\section{qRT-PCR analysis}

Total RNA was extracted from the tissues and cells using TRIzol reagent, and reverse transcription was performed using a TaqMan microRNA Reverse Transcription Kit. PCR amplification was conducted using SYBR Premix Ex Taq with U6 and GAPDH as the loading controls of miR-424-5p and FOXK1.

\section{Dual-luciferase reporter gene assay}

The binding site between miR-424-5p and FOXK1 was predicted by Jefferson. The wild type (Wt) and mutant type (Mut) FOXK1 3'UTR luciferase vectors (WT-3'UTR and MUT-3'UTR) were constructed and 
cloned into the pmiR-RB-reporter plasmid. Then, Pmir-FOXK1-WT and Pmir-FOXK1-MUT were transfected into HL-7702 cells with miR-424-5p mimic, miR-424-5p inhibitor, and miR-424-5p-NC using a Lipofectamine 2000 system for 48 h. Finally, the luciferase activity of all transfected cells was detected.

\section{Western blotting analysis}

Total protein in the cells and exosomes was extracted, sodium dodecyl sulfate-polyacrylamide gel electrophoresis was conducted, and then the samples were transferred onto membranes. The membranes were blocked with 5\% skim milk powder and incubated with primary antibodies FOXK1 (1: 1000; Abcam, MA, USA), CD63 (1: 500), and CD81 (1: 500) at $4^{\circ} \mathrm{C}$ overnight, and then with the respective secondary antibody (1: 3000, CA, USA). An enhanced chemiluminescent system was used for detection using GAPDH as the internal reference.

\section{Cell apoptosis and viability analyses}

Apoptotic BM-MSCs were detected using FCM with an Annexin-V FITC kit, according to the manufacturer's instructions. The apoptosis of the HCC cells was determined using flow cytometry, and an FC500 MCL flow cytometer was used to detect the apoptosis rate. Cell activity was analyzed using an MTT assay, as previously described and absorbance was assessed at $570 \mathrm{~nm}$ using a microplate reader (Bio-Rad Laboratories, CA, USA).

\section{Transwell assay}

The transfected cells were added into Transwell apical chambers coated with Matrigel (not used in the migration assay), while the basolateral chambers were appended with $500 \mu \mathrm{L}$ of complete medium. After $24 \mathrm{~h}$ of incubation, the cells on the microporous membrane were removed and the cells in the chambers were fixed and stained with crystal violet for $10 \mathrm{~min}$, and were then observed under an inverted microscope.

\section{Subcutaneous in vivo tumorigenesis in nude mice}

Male BALB/c nude mice were subcutaneously injected with transfected HL-7702 cells. After tumors had developed, the tumors were measured and volume was calculated using the formula: $a b^{2} / 2$ (a: length diameter, b: width diameter). The mice were euthanized on the $35^{\text {th }}$ day and the tumors were isolated and weighed. Animal experiments were conducted in strict accordance with the Guide to the Management and Use of Laboratory Animals issued by the National Institutes of Health. The mice were housed as stipulated by the protocols of animal experiments and were approved by the Institutional Animal Care and Use Committee of our Hospital.

\section{Statistical analysis}

All data analyses were conducted using SPSS 21.0 software. Measurement data that conformed to normal distribution were expressed as mean \pm standard deviation. The unpaired t-test was conducted for 
comparisons between two groups, while one-way analysis of variance (ANOVA) was used for comparisons among multiple groups and Tukey's post hoc test was used for pairwise comparisons after one-way ANOVA. A $P$ value of $<0.05$ was indicative of a statistically significant difference.

\section{Results}

\section{MiR-424-5p was downregulated in HCC tissues and cells and was associated with poor survival in HCC patients}

miR-424-5p expression in HCC and adjacent non-tumor liver tissues was determined using qRT-PCR, and the results showed that miR-424-5p was downregulated in HCC tissues, compared with adjacent nontumor liver tissues (Figure 1A). Subsequently, the expression of miR-424-5p in HCC patient tissues was determined to evaluate the prognostic value of miR-424-5p for HCC patients, and patients were divided into a high expression group $(n=49)$ and a low expression group $(n=48)$ based on their level of miR-424$5 p$ expression. The results implied that HCC patients with higher miR-424-5p expression had a much longer overall survival time, compared with HCC patients with lower miR-424-5p expression (Figure 1B). Meanwhile, miR-424-5p expression in the HCC cell lines was also detected and it was found that the HCC cell lines had lower miR-424-5p expression than the L02 cell line, while the MHCC97-H cell line had the lowest miR-424-5p expression (Figure 1C).

\section{BM-MSC derived exosomes transfer miR-424-5p into HCC cells}

BM-MSCs have been confirmed to be potential therapeutic tools for tumor treatment. BM-MSCs were isolated and subjected to flow cytometry for the detection of BM-MSC surface markers (CD44, CD90, $C D 45$, and CD34). Our results showed that the cells expressed high levels of CD90 and CD44 but were negative for CD45 and CD34 (Figure 2A). Then, the exosomes were extracted from BM-MSCs and were observed under a transmission electron microscope (TEM). We discovered that the mean diameter of the exosomes was 30-100 nm (Figure 2B). Western blot analysis indicated that the exosomes expressed exosomal markers including CD63 and CD81, which were isolated from BM-MSCs (Figure 2C). To verify whether exosomes could be taken-up by HCC cells, $\mathrm{PKH} 26$ was used for exosome labeling through coculture with the MHCC97H cells. Laser scanning confocal microscopy was used to demonstrate that the labeled exosomes were taken-up by the MHCC97H cells (Figure 2E).

Next, the expression of miR-424-5p in the transfected BM-MSC-derived exosomes was determined and the results showed that BM-MSC-derived exosomes conveying the miR-424-5p mimic upregulated miR-424$5 p$, whereas BM-MSC-derived exosomes conveying the miR-424-5p inhibitor downregulated miR-424-5p (Figure 2D). miR-424-5p expression of the MHCC97-H cells was detected, and we found that the exosomes could either upregulate or downregulate miR-424-5p expression (Figure 2F).

BM-MSC derived exosomes inhibit HCC cell proliferation and suppress motility in vitro and in vivo 
To investigate whether the BM-MSC-derived exosomes could affect $\mathrm{HCC}$ cell function, the exosomes were extracted and added to the HCC cells. Then, MTT assay, flow cytometry, and transwell assay were performed to detect any proliferative, cell cycle, apoptosis, and motility ability changes that may take place under the effect of the BM-MSC-derived exosomes. The results show that the BM-MSC-derived exosomes inhibited HCC cell proliferation, migration, and invasion, and promoted apoptosis of MHCC97$\mathrm{H}$ cells, and also arrested the cell cycle at the $\mathrm{G} 0 / \mathrm{G} 1$ phase (Figure $3 \mathrm{~A}, \mathrm{~B}, \mathrm{C}$, and $\mathrm{D}$ ). The in vivo assay results also showed that injection of BM-MSC-derived exosomes can suppress tumor volume and weight in a xenograft model (Figure 3E and F). All these results suggested that BM-MSC-derived exosomes could ameliorate the malignant activity of the HCC cells.

\section{BM-MSC-derived exosomes deliver MiR-424-5p to suppress HCC cell growth and motility in vitro and in vivo}

The results mentioned above demonstrated that BM-MSC-derived exosomes can inhibit the malignant function of HCC cells both in vitro and in vivo. Meanwhile, the downregulation of miR-424-5p was found in both HCC cell lines and HCC tissues. To further elucidate the function of miR-424-5p, we upregulated and suppressed miR-424-5p expression in exosomes and delivered them into HCC cells, and then reevaluated cell function.

The results of the MTT assay, flow cytometry, and transwell assay also confirmed that the BM-MSCderived exosomes that transferred the miR-424-5p mimic restrained proliferation, migration, and invasion, and promoted the apoptosis of MHCC97-H cells and also arrested the cell cycle at the G0/G1 phase. The effects of the BM-MSC-derived exosomes that silenced miR-424-5p in HCC cells were contrary to that of the BM-MSC-derived exosomes that overexpressed miR-424-5p (Figure 4A, B, C, and D). The in vivo assay also showed that the injection of BM-MSC-derived exosomes conveying the miR-424-5p mimic repressed tumor volume and the weight of the xenografts, while BM-MSC-derived exosomes that downregulated miR-424-5p exerted an opposite impact (Figure 4E and F). Therefore, we can conclude that BM-MSCderived exosomal miR-424-5p can suppress the malignant activity of HCC cells.

\section{FOXK1 is the target gene that is negatively regulated by miR-424-5p}

The online miRNA prediction website Targetscan was used to predict the potential target of miR-424-5p. We found that miR-424-5p can bind to the 3'-UTR of FOXK1, which was confirmed through dual-luciferase reporter assay (Figure 5A and B). Next, we examined FOXK1 expression in HCC tissues and adjacent nontumor liver tissues using quantitative real-time polymerase chain reaction and western blotting analysis. The results showed that FOXK1 was significantly upregulated in HCC tissues, compared with adjacent non-tumor liver tissues (Figure $5 \mathrm{C}$ and $\mathrm{D}$ ).

We detected further changes in FOXK1 expression after the MHCC97H cells were supplemented with BMMSC-derived exosomes using quantitative real-time polymerase chain reaction and western blotting analysis. The results showed that BM-MSC-derived exosomes that overexpress miR-424-5p can downregulate FOXK1 expression, while exosomes that inhibit miR-424-5p can upregulate FOXK1 
expression in MHCC97-H cells (Figure 5E and G), demonstrating the negative regulatory relationship between miR-424-5p and FOXK1. The results of the Pearson analysis also implied the negative relationship between the expression of miR-424-5p and FOXK1 (Figure 5F).

\section{Knockdown of FOXK1 expression suppresses biological processes in HCC cells}

Since FOXK1 was overexpressed in HCC tissues and HCC cells, the specific siRNA that targeted FOXK1 was used to detect the function of FOXK1 in HCC cells in combination with the miR-424-5p inhibitor. It was found that si-FOXK1 suppressed FOXK1 gene and protein expression, inhibited the proliferation, migration, and invasion abilities of HCC cells, and promoted HCC cell apoptosis, and arrested the cell cycle at the G0/G1 phase. Furthermore, knockdown of FOXK1 simultaneously inhibited miR-424-5p expression, and this effect was reversed by the silencing of FOXK1 in HCC cells (Figure 6A, B, C, D, and E). Overall these results provide evidence indicating that miR-424 regulates $\mathrm{HCC}$ cell functions by targeting FOXK1.

\section{Discussion}

The incidence and severity of $\mathrm{HCC}$ are increasing globally, while HCC prognosis is still unsatisfactory due to high rates of recurrence and metastasis ${ }^{28,29}$. Thus, improved methods of treatment are critically required to provide a better prognosis for $\mathrm{HCC}$ patients 5,30 . This study was designed to investigate the impacts of BM-MSCs-derived exosomal miR-424-5p on the development of HCC via targeting FOXK1. We found that BM-MSC-derived exosomes upregulated miR-424-5p expression to suppress malignant phenotypes of HCC cells by inhibiting FOXK1, thus restraining HCC progression.

First, we determined miR-424-5p and FOXK1 expression levels in tissues and cells and found that miR424-5p expression was downregulated whereas FOXK1 expression was upregulated in HCC tissues and cells, compared with adjacent normal tissues and the human normal hepatocyte line, L02. Similarly, it was previously found that miR-424-5p expression is downregulated in HCC tissues, cell lines ${ }^{31}$, and detached anoikis-resistant HCC cells ${ }^{32}$. A recent study revealed that FOXK1 is upregulated in human HCC tissue and is positively associated with cancer progression ${ }^{22,}{ }^{23}$. Moreover, miR-424-5p expression in BMMSC-derived exosomes was also detected. The results showed that BM-MSC-derived exosomes were able to upregulate miR-424-5p expression. Delivery of miR-424-5p via exosomes can promote triple-negative breast cancer cell apoptosis ${ }^{33}$. However, the expression of BM-MSC-derived exosomal miR-424-5p in HCC is still unexplored. Additionally, we evaluated the relationship between miR-424-5p and FOXK1 in HCC, and it was confirmed through a bioinformatics method and dual-luciferase reporter gene assay that FOXK1 is a target gene of miR-424-5p. Nevertheless, this relationship has rarely been studied.

The HCC cells were co-cultured with BM-MSC-derived exosomes to determine the effect of the exosomes on the biological processes of HCC cells, and we found that BM-MSC-derived exosomes suppressed the proliferation, migration, invasion, in vivo tumorigenesis, and promoted the apoptosis of HCC cells. Similar to our findings, BM-MSC-derived exosomes delivered miR-124 to inhibit pancreatic cancer cell 
proliferation, epithelial-mesenchymal transition, and enhanced chemotherapy sensitivity ${ }^{34}$. BM-MSCderived exosomes alleviated radiation-induced bone loss by activating Wnt/ $\beta$-catenin signaling ${ }^{35}$. In another study, it was demonstrated that BM-MSC-derived exosomes could inhibit the viability, migration, and invasion of bladder cancer cells ${ }^{36}$. It has been also been shown that hypoxia-elicited MSC-derived exosomes can facilitate cardiac repair through miR-125b-mediated prevention of cell death in myocardial infarction ${ }^{37}$.

In our study, HCC cells with abnormal miR-424-5p expression were treated with transfected BM-MSCderived exosomes to determine the effect of BM-MSCs-derived exosomal miR-424-5p on HCC cells. Our results indicated that miR-424-5p overexpression suppressed the malignant behaviors of HCC cells. Consistently, another study reported that miR-424-5p upregulation inhibited HCC cell proliferation, invasion, and tumor growth ${ }^{38}$, suggesting that miR-424-5p reversed resistance to anoikis, the epithelialmesenchymal transition of HCC cells, and significantly repressed the tumorigenicity of HCC cells ${ }^{32}$.

Based on the targeted relationship between miR-424-5p and FOXK1, we knocked-down FOXK1 to investigate its impact on HCC cells. The results showed that FOXK1 silencing inhibited the proliferation, migration, invasion, and in vivo tumorigenesis, but promoted the apoptosis of HCC cells. Similarly, other studies have also reported that the downregulation of FOXK1 restricts HCC cell growth ${ }^{23}$, and have elucidated that FOXK1 silencing can repress the viability of liver cancer cells by modulating glycolysis ${ }^{39}$, 40. mTORC1 promotes metabolic reprogramming by suppressing GSK3-dependent FOXK1 phosphorylation ${ }^{41}$. Additionally, we validated that FOXK1 downregulation was capable of reversing the effects of the miR-424-5p inhibitor on the biological processes of HCC cells. Consistently, FOXK1 upregulation has been verified to reverse the effect of miR-485-5p upregulation on the proliferation, migration, invasion, and apoptosis of colorectal cancer cells ${ }^{42}$. It has also been shown that that FOXK1 overexpression can restore repressed lung cancer cell proliferation and metastasis induced by the miR195-5p mimic ${ }^{43}$.

In summary, our results revealed that BM-MSC-derived exosomes can upregulate miR-424-5p expression to restrict malignant phenotypes of HCC cells by inhibiting FOXK1, thus restraining HCC progression. This study may provide potential biomarkers for HCC diagnosis and treatment. However, further exploration of the molecular mechanisms involved in HCC is needed.

\section{Declarations}

\section{Author's contributions}

Jikui Liu conceived the study; Zewei Lin and Qingqi Ren performed the experiment; Xiaofei Ma conducted the statistical analysis; Zewei Lin wrote the manuscript. All authors have read and approved the final manuscript.

\section{Funding}


This study was supported by Sanming Project of Medicine in Shenzhen (No. SZSM201612021), Special Fundation for Science and Technology Development of Guangdong Province (No. 2017B090904010), Scientific Research Project of Health and Family Planning Commission of Shenzhen Municipality (No. SZXJ2018086); Science Technology and Innovation Commission of Shenzhen Municipality (No. JCYJ20190809095801653, JCYJ20190809100217290);

\section{Compliance with ethical standards}

\section{Conflict of interest}

The authors declare that they have no conflict of interest.

\section{Ethics approval and consent to participate}

This study was approval by the Ethics Committee of Shenzhen Hospital, Peking University and informed consent was obtained from each patient. All animal studies were conducted with the approval from the Institutional Animal Care and Use Committee of Shenzhen Hospital, Peking University

\section{References}

1. Sung H, Ferlay J, Siegel RL, et al. Global cancer statistics 2020: GLOBOCAN estimates of incidence and mortality worldwide for 36 cancers in 185 countries. CA Cancer J Clin 2021.

2. Siegel RL, Miller KD, Fuchs HE, et al. Cancer Statistics 2021 CA Cancer J Clin. 2021;71:7-33.

3. Huang DQ, El-Serag HB, Loomba R. Global epidemiology of NAFLD-related HCC: trends, predictions, risk factors and prevention. Nat Rev Gastroenterol Hepatol 2020.

4. Yang JD, Heimbach JK. New advances in the diagnosis and management of hepatocellular carcinoma. BMJ. 2020;371:m3544.

5. Llovet JM, De Baere T, Kulik L, et al. Locoregional therapies in the era of molecular and immune treatments for hepatocellular carcinoma. Nat Rev Gastroenterol Hepatol 2021.

6. Rimassa L, Personeni N, Czauderna C, et al. Systemic treatment of HCC in special populations. J Hepatol. 2021;74:931-43.

7. Petrowsky H, Fritsch R, Guckenberger M, et al. Modern therapeutic approaches for the treatment of malignant liver tumours. Nat Rev Gastroenterol Hepatol. 2020;17:755-72.

8. Levy 0 , Kuai R, Siren EMJ, et al. Shattering barriers toward clinically meaningful MSC therapies. Sci Adv. 2020;6:eaba6884.

9. Song N, Scholtemeijer M, Shah K. Mesenchymal Stem Cell Immunomodulation: Mechanisms and Therapeutic Potential. Trends Pharmacol Sci. 2020;41:653-64.

10. Thietart S, Rautou PE. Extracellular vesicles as biomarkers in liver diseases: A clinician's point of view. J Hepatol. 2020;73:1507-25. 
11. Dai J, Su Y, Zhong S, et al. Exosomes: key players in cancer and potential therapeutic strategy. Signal Transduct Target Ther. 2020;5:145.

12. Rahmani A, Saleki K, Javanmehr N, et al. Mesenchymal stem cell-derived extracellular vesicle-based therapies protect against coupled degeneration of the central nervous and vascular systems in stroke. Ageing Res Rev. 2020;62:101106.

13. Klingenberg $M$, Matsuda $A$, Diederichs $S$, et al. Non-coding RNA in hepatocellular carcinoma: Mechanisms, biomarkers and therapeutic targets. J Hepatol. 2017;67:603-18.

14. Wang X, He Y, Mackowiak B, et al. MicroRNAs as regulators, biomarkers and therapeutic targets in liver diseases. Gut. 2021;70:784-95.

15. Komoll RM, Hu Q, Olarewaju O, et al. MicroRNA-342-3p is a potent tumour suppressor in hepatocellular carcinoma. J Hepatol. 2021;74:122-34.

16. Liu Y, Lu LL, Wen D, et al. MiR-612 regulates invadopodia of hepatocellular carcinoma by HADHAmediated lipid reprogramming. J Hematol Oncol. 2020;13:12.

17. Xu WP, Liu JP, Feng JF, et al. miR-541 potentiates the response of human hepatocellular carcinoma to sorafenib treatment by inhibiting autophagy. Gut. 2020;69:1309-21.

18. Teng F, Zhang JX, Chang QM, et al. LncRNA MYLK-AS1 facilitates tumor progression and angiogenesis by targeting miR-424-5p/E2F7 axis and activating VEGFR-2 signaling pathway in hepatocellular carcinoma. J Exp Clin Cancer Res. 2020;39:235.

19. Tang M, Feng X, Pei G, et al. FOXK1 Participates in DNA Damage Response by Controlling 53BP1 Function. Cell Rep. 2020;32:108018.

20. Liu Y, Ding W, Ge H, et al. FOXK transcription factors: Regulation and critical role in cancer. Cancer Lett. 2019;458:1-12.

21. Sukonina $\mathrm{V}, \mathrm{Ma} \mathrm{H}$, Zhang W, et al. FOXK1 and FOXK2 regulate aerobic glycolysis. Nature. 2019;566:279-83.

22. Cao H, Chu X, Wang Z, et al. High FOXK1 expression correlates with poor outcomes in hepatocellular carcinoma and regulates stemness of hepatocellular carcinoma cells. Life Sci. 2019;228:128-34.

23. Li P, Yu Z, He L, et al. Knockdown of FOXK1 inhibited the proliferation, migration and invasion in hepatocellular carcinoma cells. Biomed Pharmacother. 2017;92:270-6.

24. Hara A, Kobayashi H, Asai N, et al. Roles of the Mesenchymal Stromal/Stem Cell Marker Meflin in Cardiac Tissue Repair and the Development of Diastolic Dysfunction. Circ Res. 2019;125:414-30.

25. Zhai M, Zhu Y, Yang M, et al. Human Mesenchymal Stem Cell Derived Exosomes Enhance Cell-Free Bone Regeneration by Altering Their miRNAs Profiles. Adv Sci (Weinh). 2020;7:2001334.

26. Fang SB, Zhang HY, Wang $C$, et al. Small extracellular vesicles derived from human mesenchymal stromal cells prevent group 2 innate lymphoid cell-dominant allergic airway inflammation through delivery of miR-146a-5p. J Extracell Vesicles. 2020;9:1723260.

27. Elahi FM, Farwell DG, Nolta JA, et al. Preclinical translation of exosomes derived from mesenchymal stem/stromal cells. Stem Cells. 2020;38:15-21. 
28. Wong $\mathrm{CM}$, Tsang FH, Ng IO. Non-coding RNAs in hepatocellular carcinoma: molecular functions and pathological implications. Nat Rev Gastroenterol Hepatol. 2018;15:137-51.

29. Giannelli G, Koudelkova P, Dituri F, et al. Role of epithelial to mesenchymal transition in hepatocellular carcinoma. J Hepatol. 2016;65:798-808.

30. Gordan JD, Kennedy EB, Abou-Alfa GK, et al. Systemic Therapy for Advanced Hepatocellular Carcinoma: ASCO Guideline. J Clin Oncol. 2020;38:4317-45.

31. Du H, Xu Q, Xiao S, et al. MicroRNA-424-5p acts as a potential biomarker and inhibits proliferation and invasion in hepatocellular carcinoma by targeting TRIM29. Life Sci. 2019;224:1-11.

32. Zhang Y, Li T, Guo P, et al. MiR-424-5p reversed epithelial-mesenchymal transition of anchorageindependent HCC cells by directly targeting ICAT and suppressed HCC progression. Sci Rep. 2014;4:6248.

33. Zhou Y, Yamamoto Y, Takeshita F, et al. Delivery of miR-424-5p via Extracellular Vesicles Promotes the Apoptosis of MDA-MB-231 TNBC Cells in the Tumor Microenvironment. Int J Mol Sci 2021;22.

34. Xu Y, Liu N, Wei Y, et al. Anticancer effects of miR-124 delivered by BM-MSC derived exosomes on cell proliferation, epithelial mesenchymal transition, and chemotherapy sensitivity of pancreatic cancer cells. Aging. 2020;12:19660-76.

35. Zuo R, Liu M, Wang Y, et al. BM-MSC-derived exosomes alleviate radiation-induced bone loss by restoring the function of recipient BM-MSCs and activating Wnt/beta-catenin signaling. Stem Cell Res Ther. 2019;10:30.

36. Cai H, Yang X, Gao Y, et al. Exosomal MicroRNA-9-3p Secreted from BMSCs Downregulates ESM1 to Suppress the Development of Bladder Cancer. Mol Ther Nucleic Acids. 2019;18:787-800.

37. Zhu LP, Tian T, Wang JY, et al. Hypoxia-elicited mesenchymal stem cell-derived exosomes facilitates cardiac repair through miR-125b-mediated prevention of cell death in myocardial infarction. Theranostics. 2018;8:6163-77.

38. Shen X, Li Y, He F, et al. LncRNA CDKN2B-AS1 Promotes Cell Viability, Migration, and Invasion of Hepatocellular Carcinoma via Sponging miR-424-5p. Cancer Manag Res. 2020;12:6807-19.

39. Sun $\mathrm{H}$, Wang $\mathrm{H}$, Wang $\mathrm{X}$, et al. Aurora-A/SOX8/FOXK1 signaling axis promotes chemoresistance via suppression of cell senescence and induction of glucose metabolism in ovarian cancer organoids and cells. Theranostics. 2020;10:6928-45.

40. Cui H, Gao Q, Zhang L, et al. Knockdown of FOXK1 suppresses liver cancer cell viability by inhibiting glycolysis. Life Sci. 2018;213:66-73.

41. He L, Gomes AP, Wang X, et al. mTORC1 Promotes Metabolic Reprogramming by the Suppression of GSK3-Dependent Foxk1 Phosphorylation. Mol Cell 2018;70:949-60 e4.

42. Liu J, Zhang J, Wang Z, et al. Knockdown of circAPLP2 Inhibits Progression of Colorectal Cancer by Regulating miR-485-5p/FOXK1 Axis. Cancer Biother Radiopharm 2020.

43. Long Z, Wang Y. miR-195-5p Suppresses Lung Cancer Cell Proliferation, Migration, and Invasion Via FOXK1. Technol Cancer Res Treat. 2020;19:1533033820922587. 
Figures
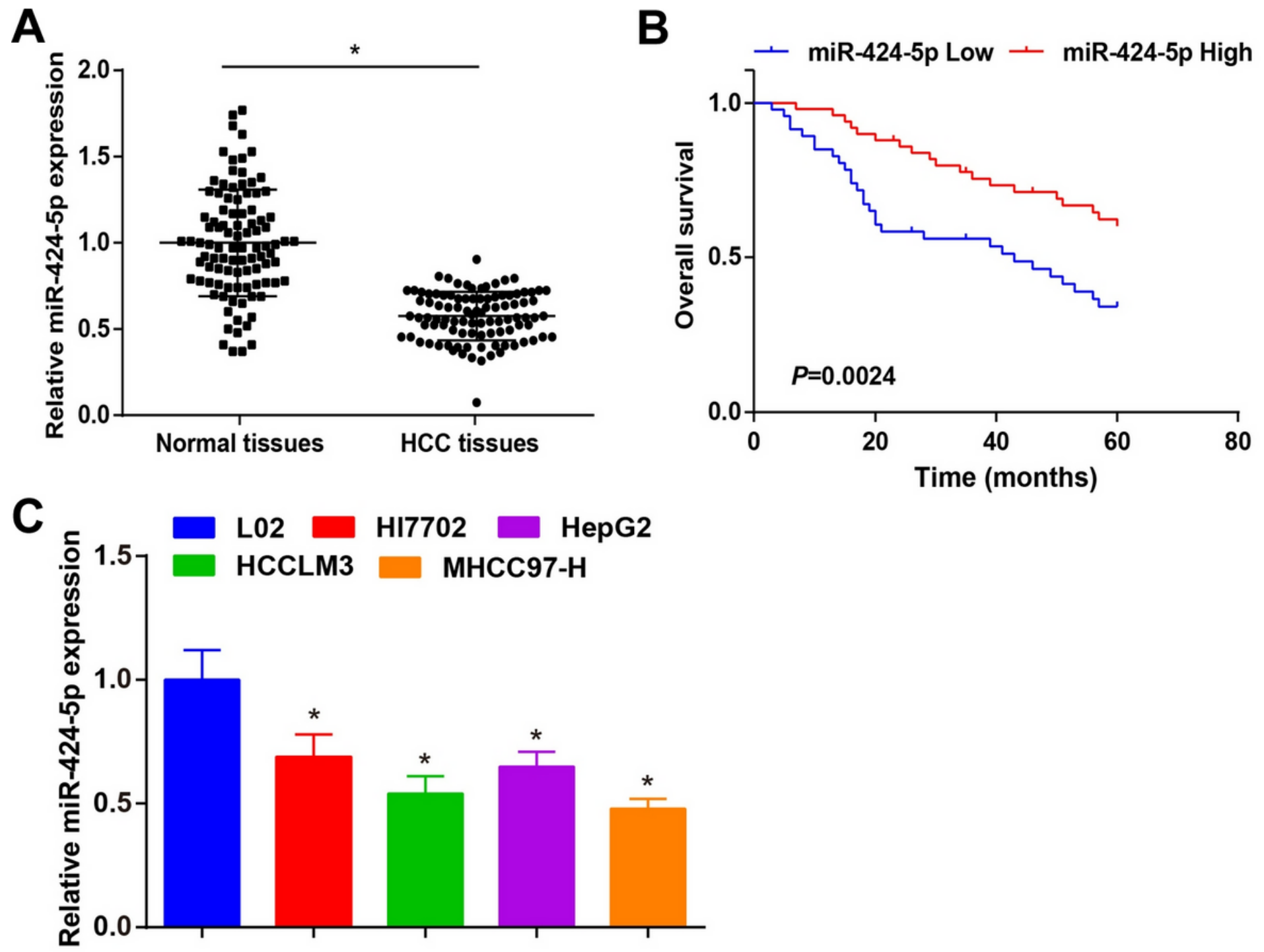

Figure 1

MiR-424-5p is downregulated in HCC tissues and cells and is associated with poor survival of HCC patients. (A) qRT-PCR was used to examine the expression of miR-424-5p in HCC and adjacent non-tumor liver tissues. (B) The correlation between miR-424-5p expression and overall survival of the $97 \mathrm{HCC}$ patients was analyzed using Kaplan-Meier analysis. (C) miR-424-5p expression was detected in HCC cell lines and human immortalized hepatocytes. ${ }^{*}, \mathrm{P}<0.05$. 
A
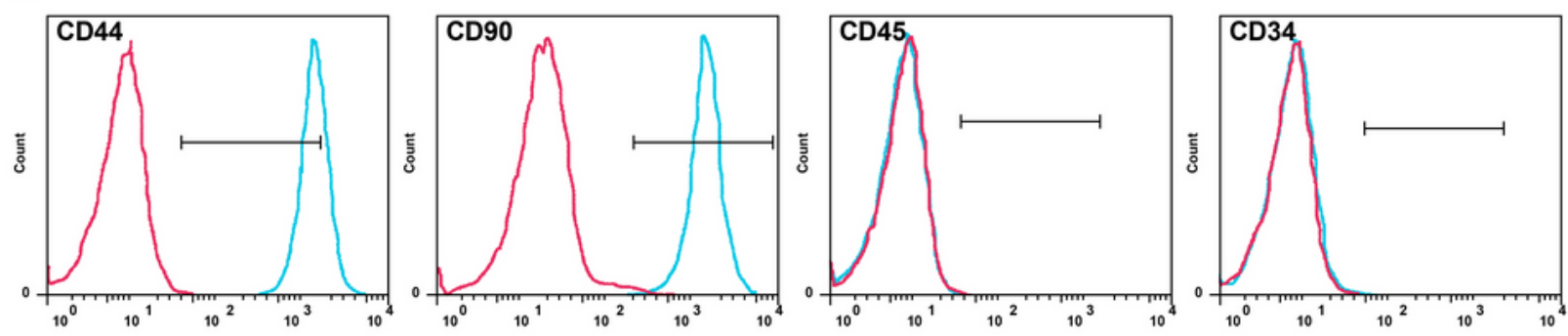

B

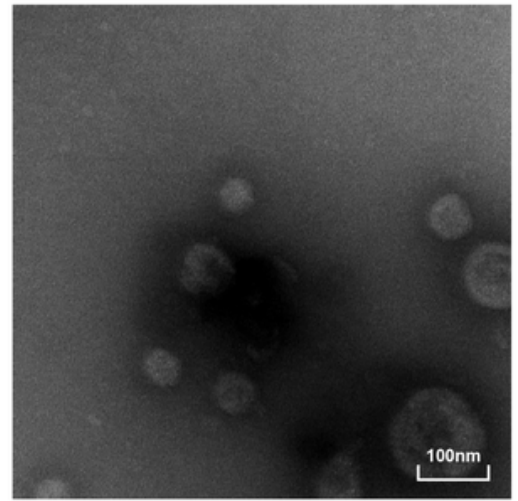

C

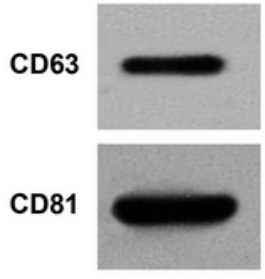

PKH26

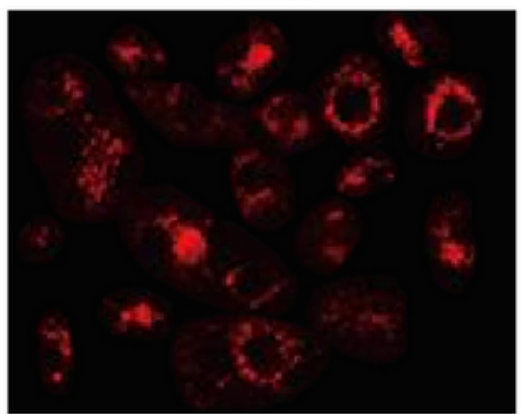

E

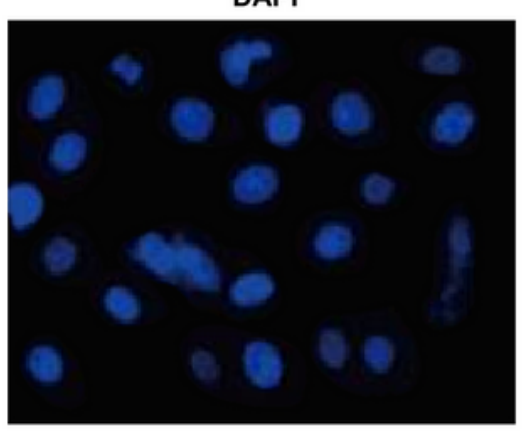

$\mathbf{F}$
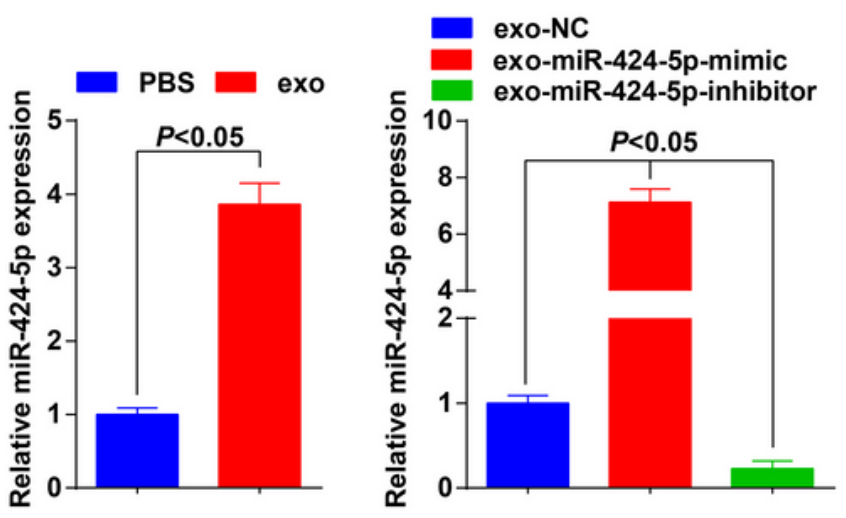

D
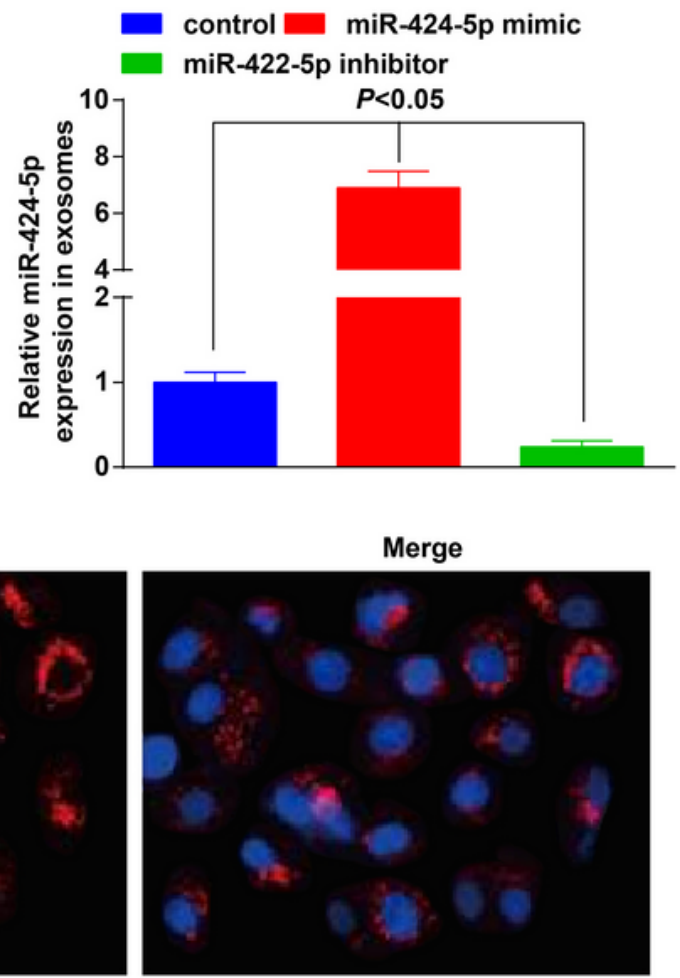

Figure 2

BM-MSC-derived exosomes were used to transfer miR-424-5p into HCC cells. (A) The identity of the BMMSCs was confirmed using flow cytometry; (B) TEM observation of exosomes; (C) exosomes were identified using western blotting analysis; (D) miR-424-5p expression in the exosomes; (E) uptake of exosomes by MHCC97-H cells; (F) miR-424-5p expression in MHCC97-H cells co-cultured with exosomes. 
A

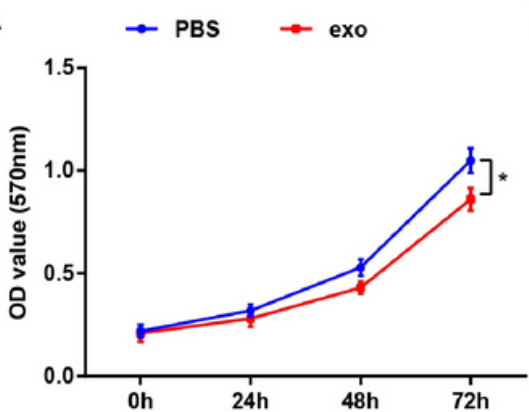

C
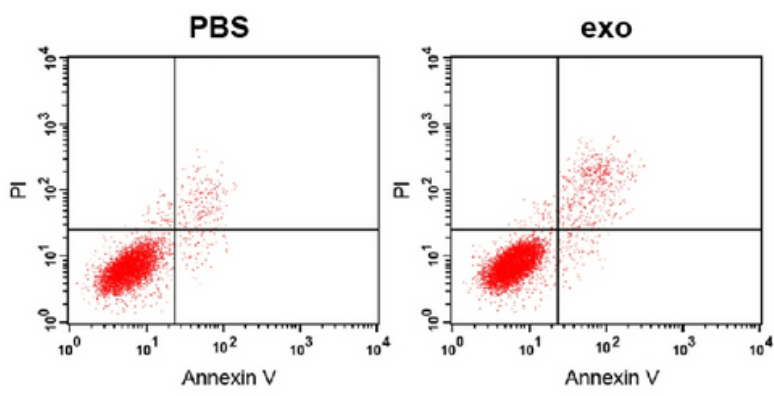

D
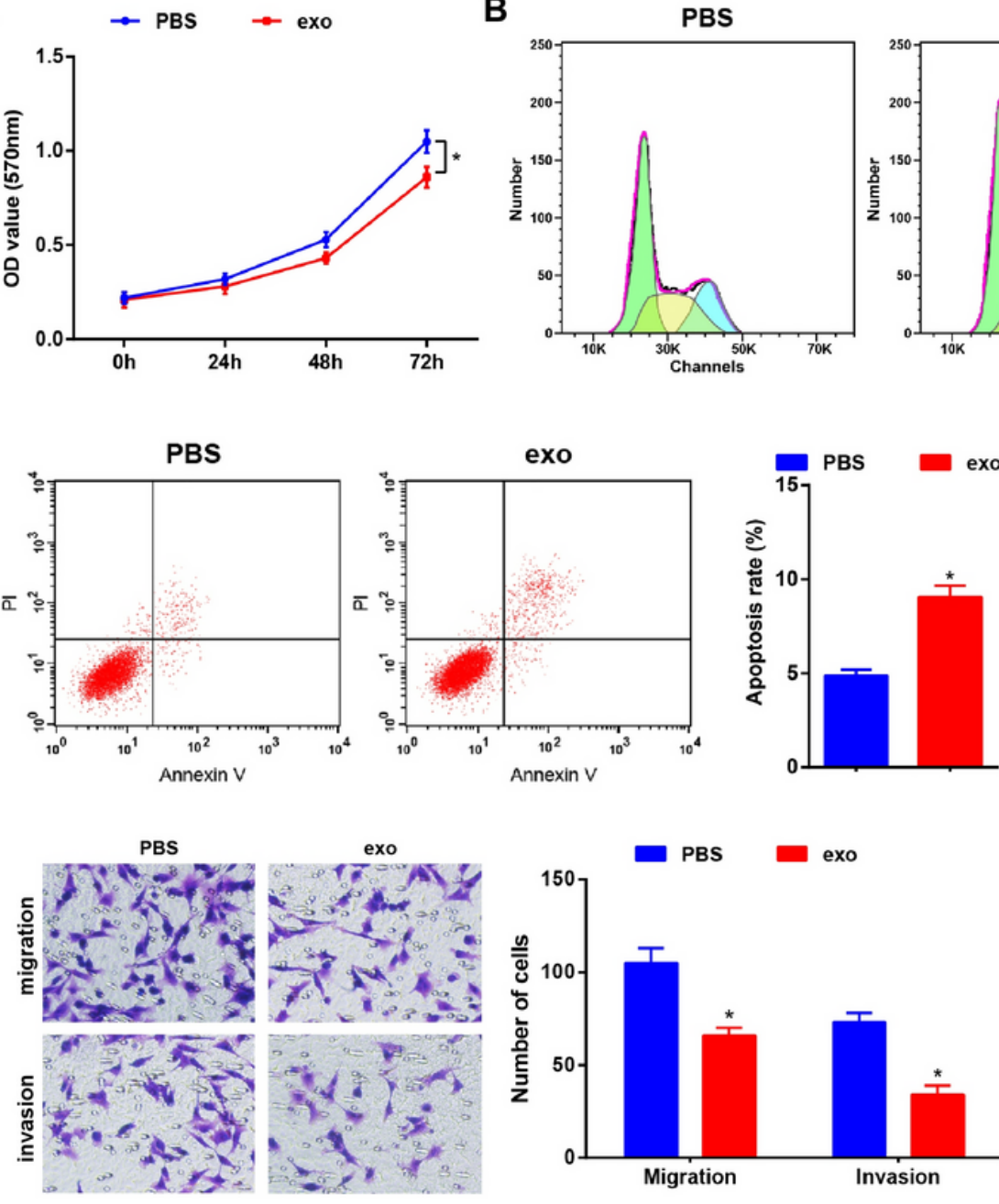

exo

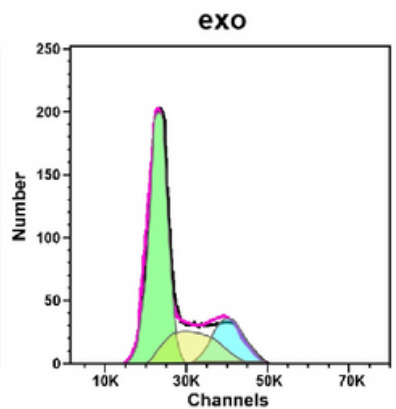

E
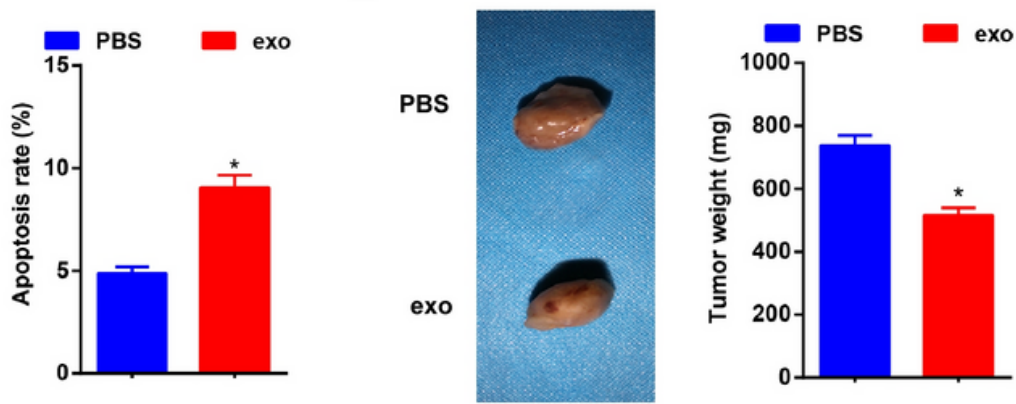

$\mathbf{F}$

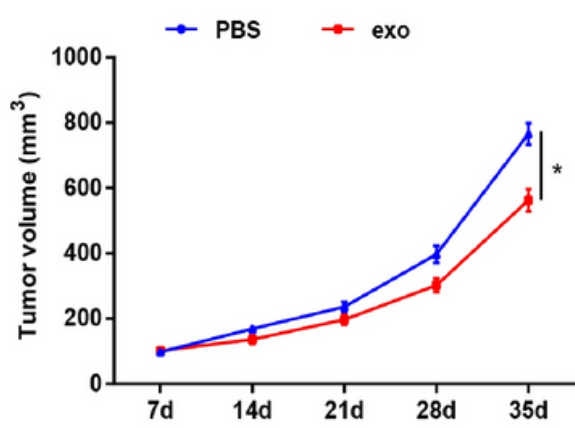

Figure 3

BM-MSC-derived exosomes inhibited proliferation and motility but promoted the apoptosis of HCC cells. (A) the proliferation of MHCC97-H cells detected using MTT assay; (B) cell cycle distribution of the MHCC97-H cells using flow cytometry; (C) apoptosis of MHCC97-H cells detected using flow cytometry; (D) migration and invasion of MHCC97-H cells detected using Transwell assay; (E) volume of xenografts from the nude mice; $(F)$ weight and representative images of the xenografts from the nude mice. 
A

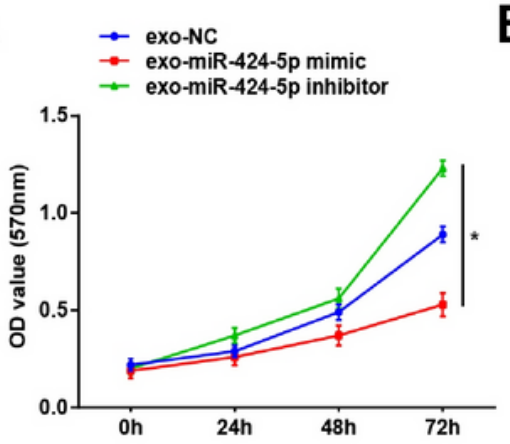

C
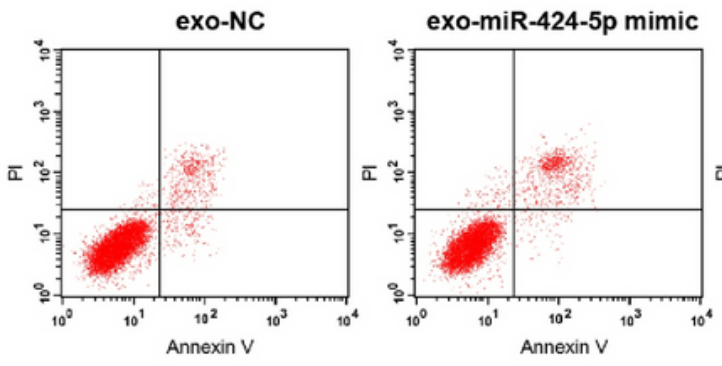

D

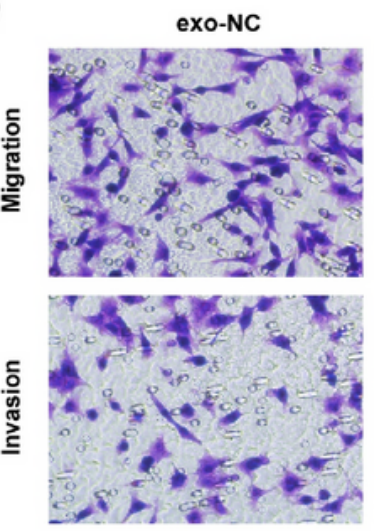

$\mathbf{E}$
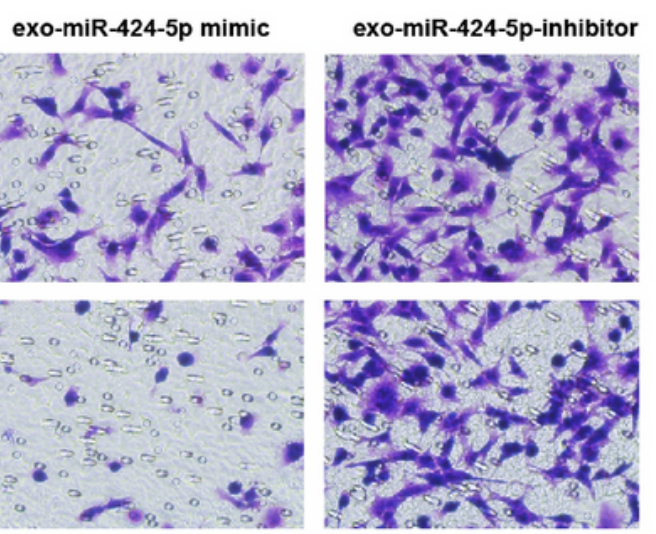

$\mathbf{F}$

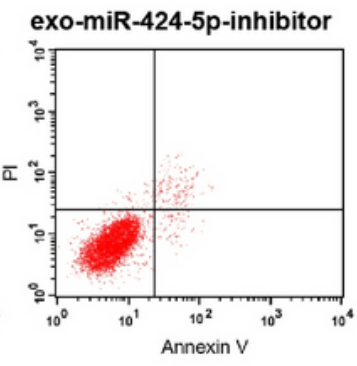

B
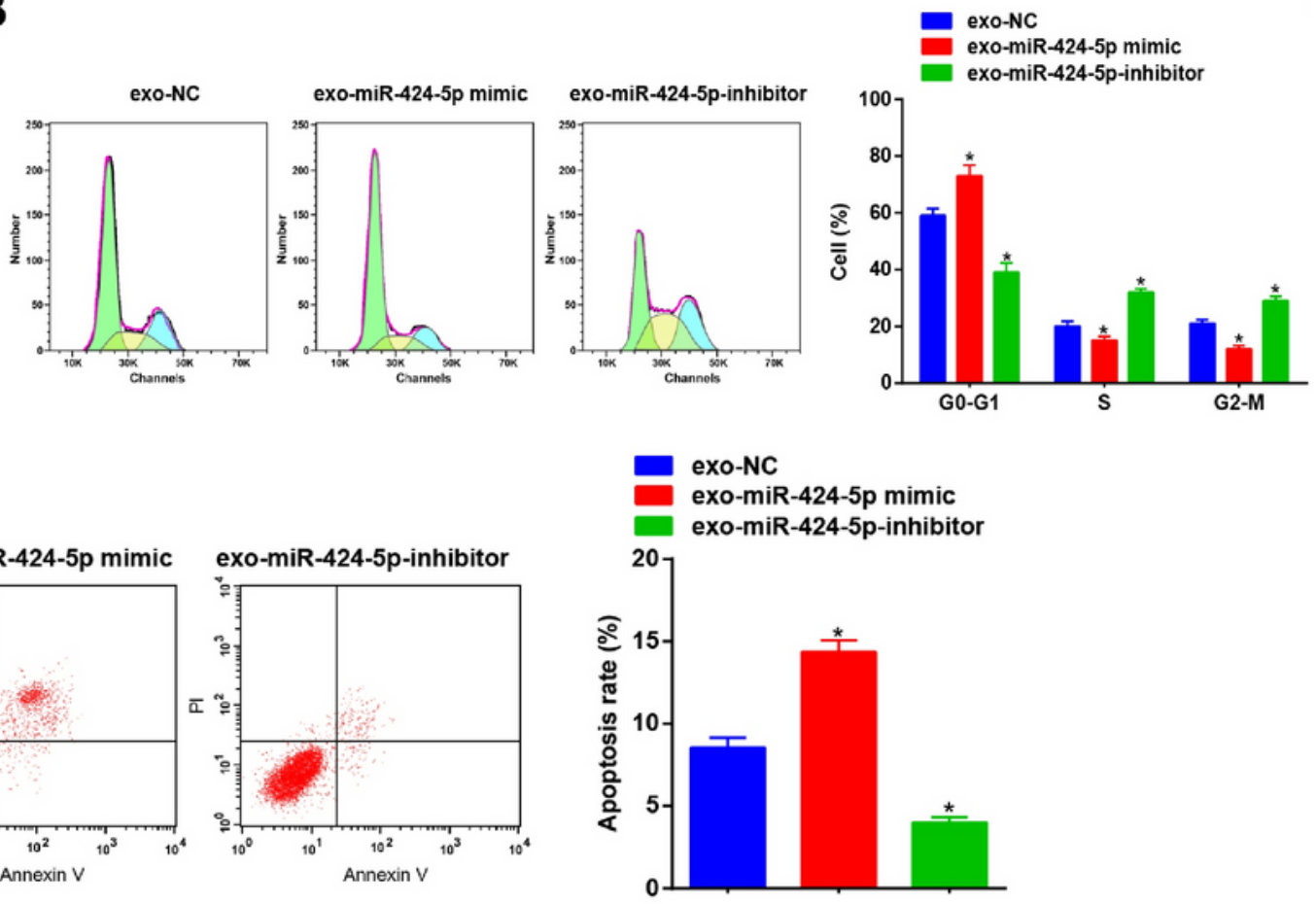
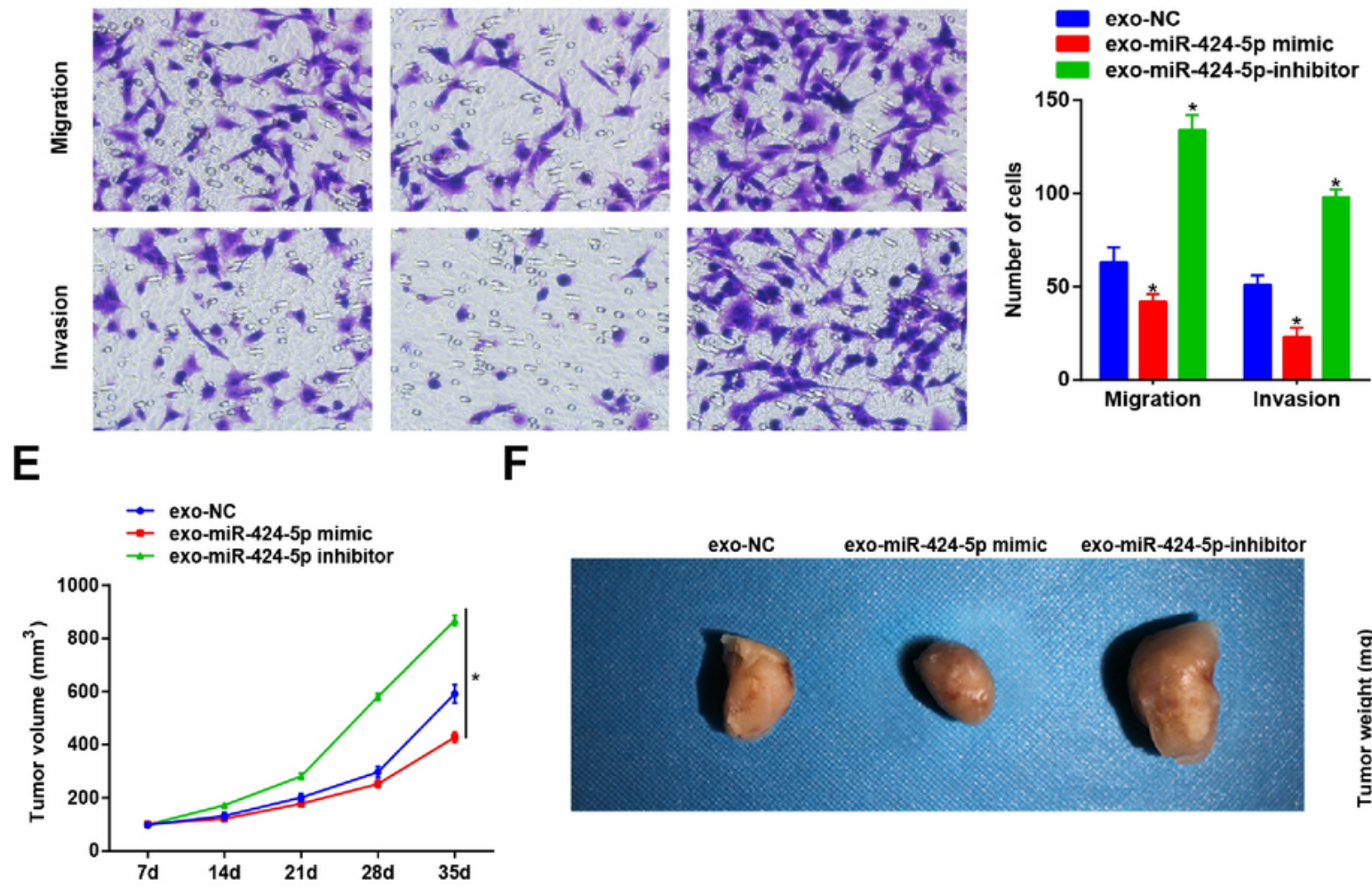

\section{Figure 4}

BM-MSC-derived exosomes delivered miR-424-5p that suppresses HCC cell growth and motility. (A) the proliferation of MHCC97-H cells detected using MTT assay; (B) cell cycle distribution of the MHCC97-H cells detected using flow cytometry; (C) apoptosis of MHCC97-H cells detected using flow cytometry; (D) migration and invasion of MHCC97-H cells detected using Transwell assay; (E) volume of the xenografts from the nude mice; (F) weight and representative images of the xenografts from the nude mice. 
A

FOXK1 3'UTR WT 5'-UACAUUGCUCACAUGUGCUGCUA-3'

miR-424-5p 3'-AAGUUUUGUACUUAACGACGAC-5'

FOXK1 3'UTR Mut 5'-UAAUGUUAUAUCUGAUAGAUUCA-3'

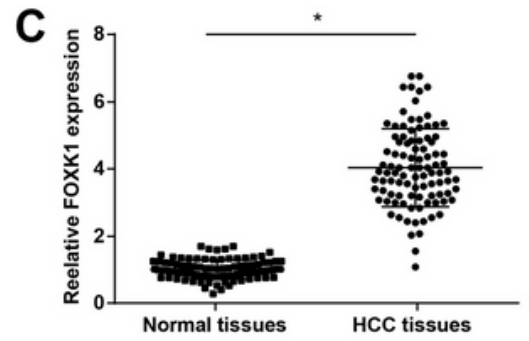

B

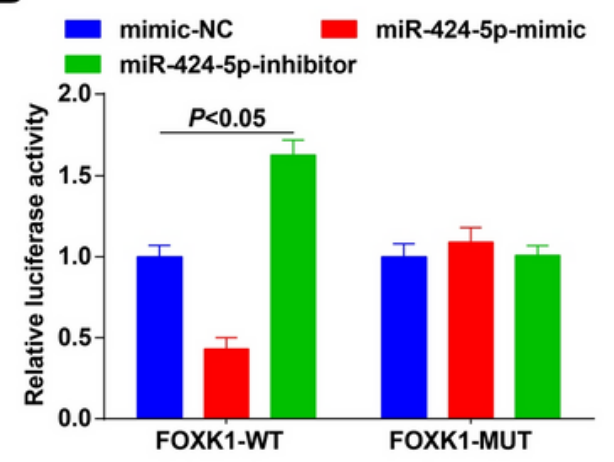

E

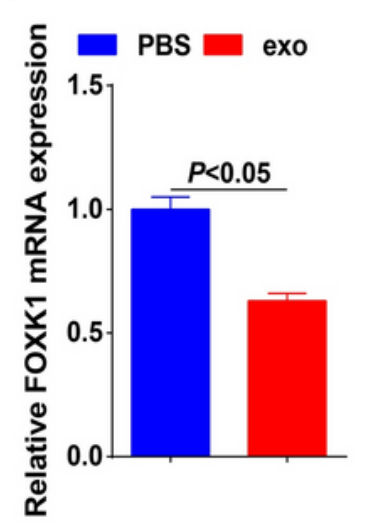

G
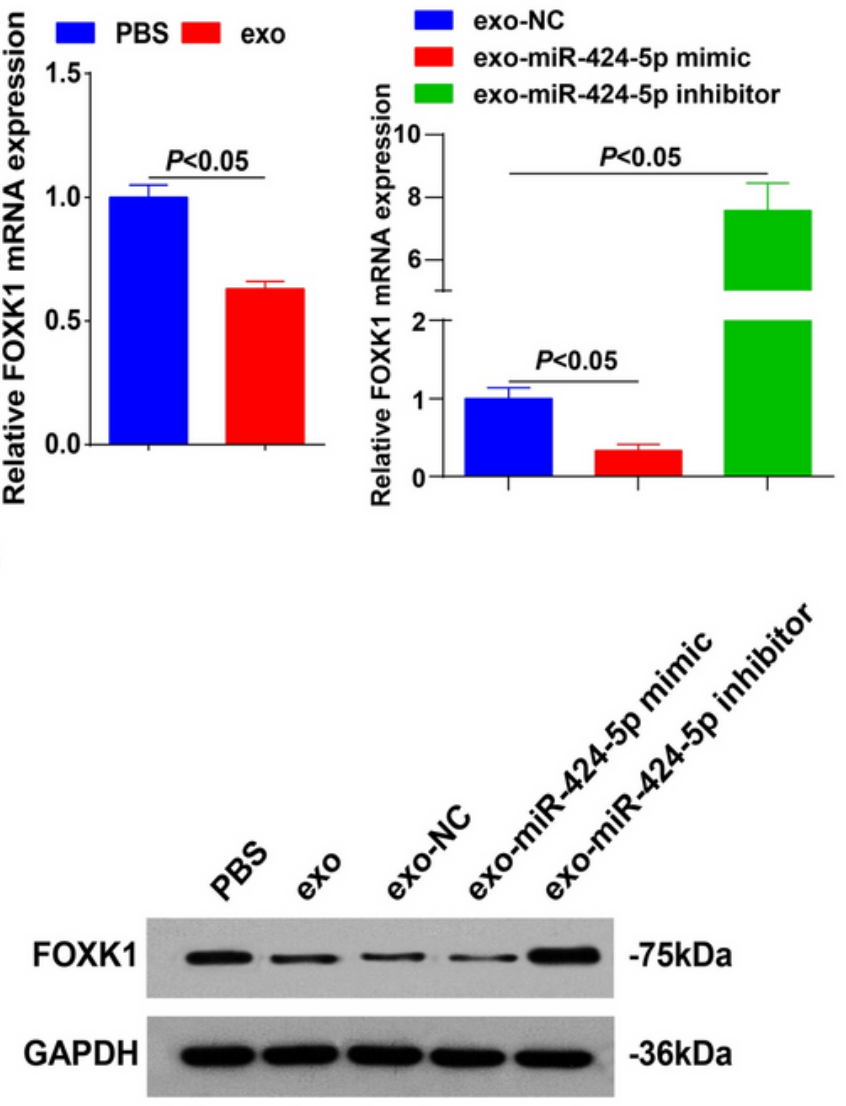

D
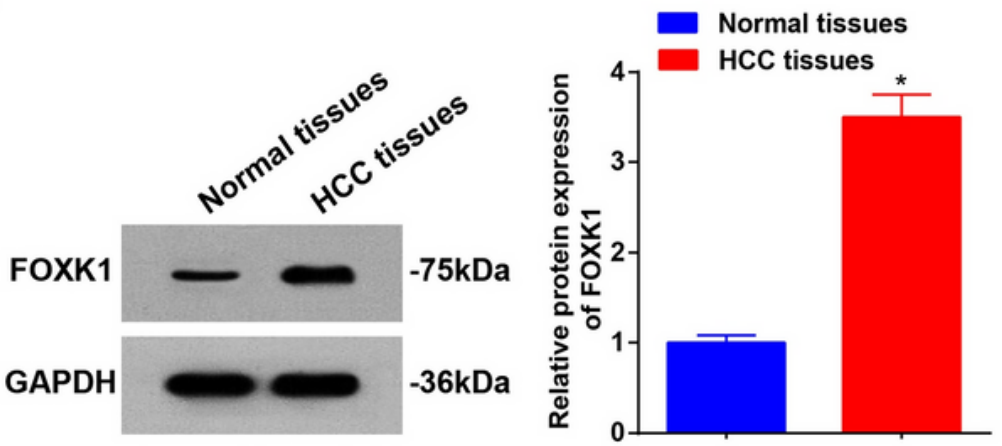

$\mathbf{F}$

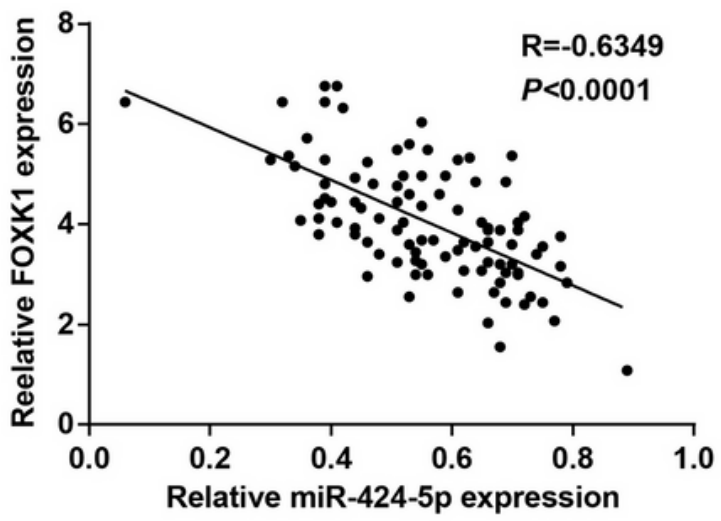

exo-miR-424-5p mimic

드

exo-miR-424-5p inhibitor

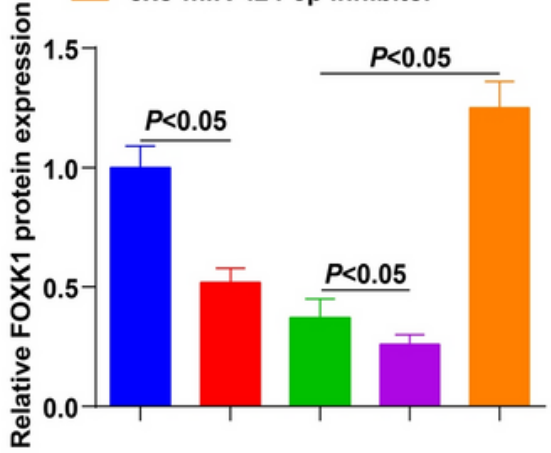

Figure 5

MiR-424-5p targets FOXK1. (A) binding sites between miR-424-5p and FOXK1; (B) the targeting relationship between miR-424-5p and FOXK1 was confirmed using dual-luciferase reporter gene assay; (C) FOXK1 expression in HCC and adjacent normal tissues; (D) protein expression of FOXK1 in HCC and adjacent normal tissues; (E) FOXK1 expression in MHCC97-H cells; (F) correlation between the expression of miR-424-5p and FOXK1 in HCC tissues; $(G)$ protein expression of FOXK1 in MHCC97-H cells. 
A

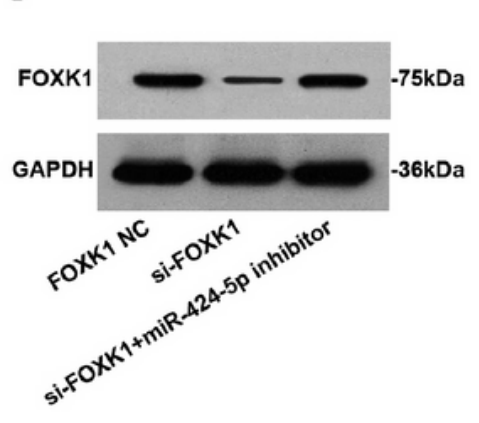

B

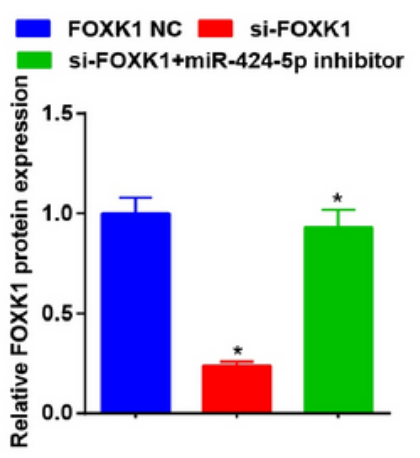

$\rightarrow$ FOXK1 NC $\rightarrow$ si-FOXK1

24-5p inhibitor

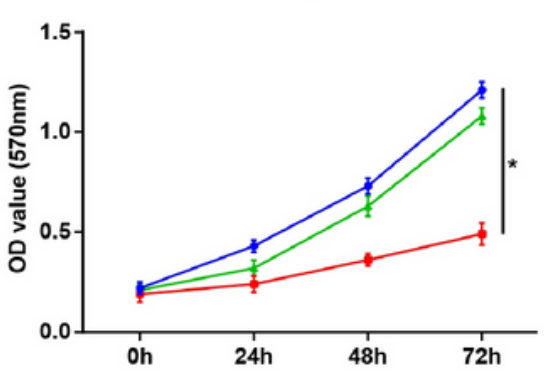

C
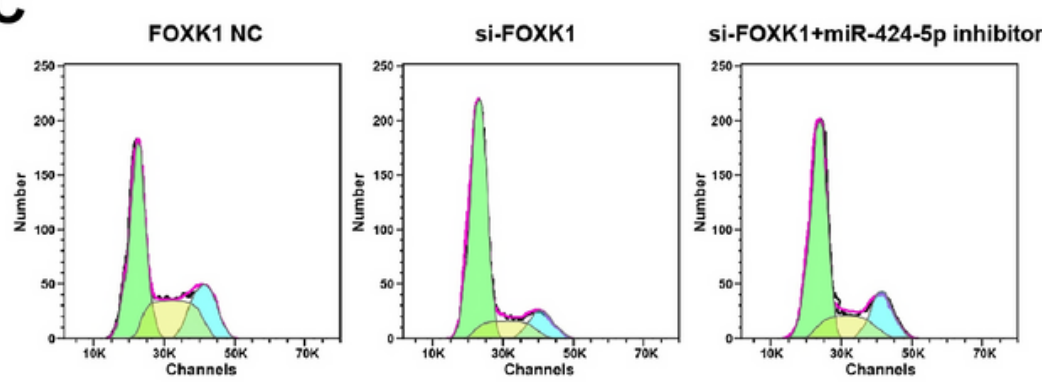

FOXK1 NC si-FOXK1 si-FOXK1+miR-424-5p inhibitor

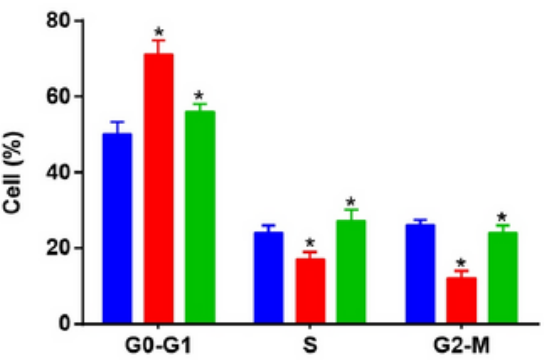

D
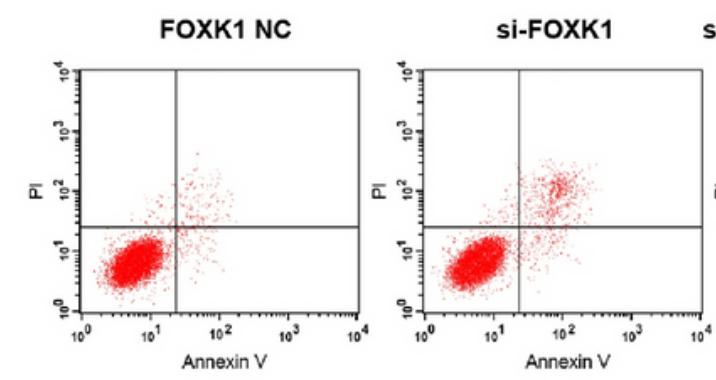

si-FOXK1+miR-424-5p inhibitor

FOXK1 NC si-FOXK1 si-FOXK1+miR-424-5p inhibitor
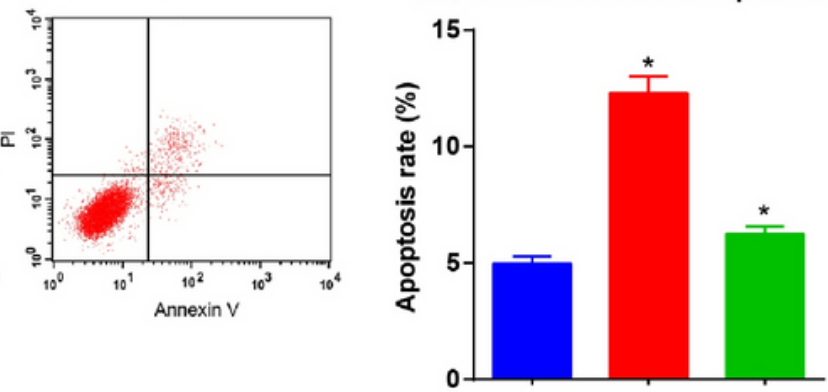

E
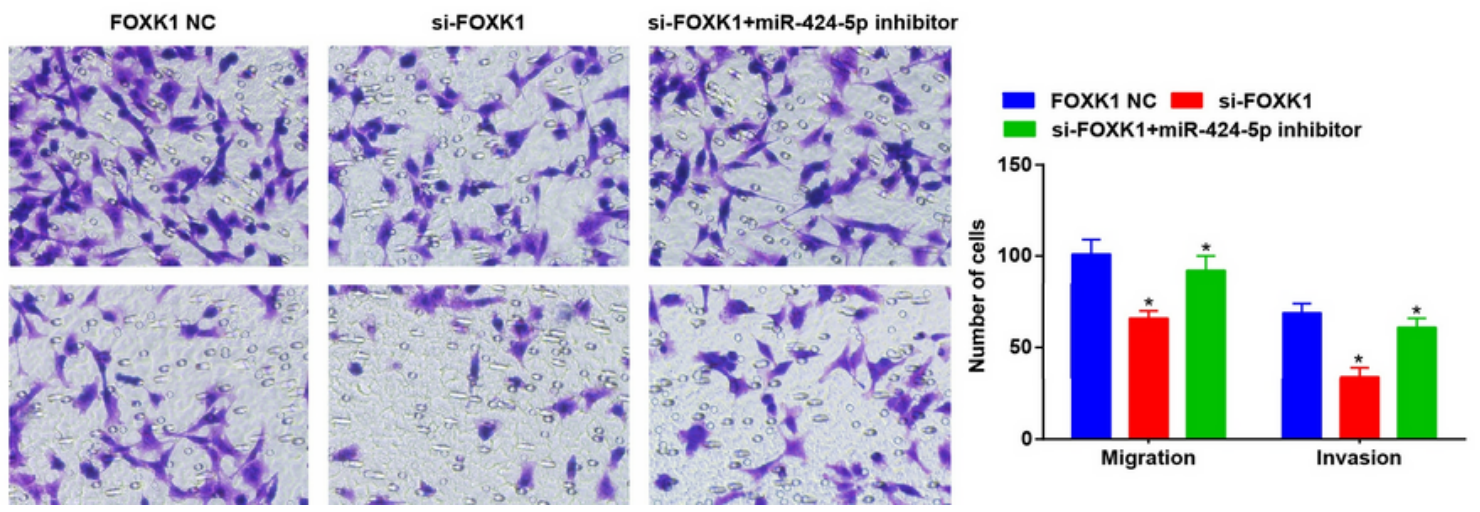

Figure 6

FOXK1 inhibition suppresses the biological processes of HCC cells. (A) protein expression of FOXK1 in MHCC97-H cells; (B) proliferation of MHCC97-H cells detected using MTT assay; (C) cell cycle distribution of MHCC97-H cells detected using flow cytometry; (D) apoptosis of MHCC97-H cells detected using flow cytometry; (E) migration and invasion of MHCC97-H cells detected using transwell assay. 